EPJ Web of Conferences 107, 10001 (2016)

DOI: $10.1051 /$ epjconf/201610710001

(C) Owned by the authors, published by EDP Sciences, 2016

\title{
Exotic nuclear matter
}

\author{
H. Lenske ${ }^{1,2, a}$, M. Dhar ${ }^{1}$, N. Tsoneva ${ }^{1,3, b}$, and J. Wilhelm ${ }^{1}$ \\ ${ }^{1}$ Institut für Theoretische Physik, Universität Giessen, Giessen, Germany \\ ${ }^{2}$ GSI Darmstadt, Darmstadt, Germany \\ ${ }^{3}$ FIAS Frankfurt Institute for Advanced Studies, Frankfurt, Germany
}

\begin{abstract}
Recent developments of nuclear structure theory for exotic nuclei are addressed. The inclusion of hyperons and nucleon resonances is discussed. Nuclear multipole response functions, hyperon interactions in infinite matter and in neutron stars and theoretical aspects of excitations of nucleon resonances in nuclei are discussed.
\end{abstract}

\section{Introduction}

In recent years, nuclear physics has undergone a rapid change by redirecting the subject from the well studied stable nuclei to the extremes of nuclear matter. Driven by a series of new experiments, nuclear theory has been refocused to investigations of asymmetric nuclear matter far off beta-stability. The isospin sector as the main working area of traditional nuclear physics has been left, extending the field into new regions now including hyperons and, as a late development, excitations of nucleon resonances as sub-nuclear degrees of freedom are being investigated. In recent years nuclear density functional theory and nuclear many-body methods - ranging from HFB and (Q)RPA theory to the QPM approach of Soloviev et al. - have been used to study systematically ground state properties and multipole response functions of exotic nuclei, especially in the region of the $N=82$ and the $Z=50$ shell closures, see e.g. [1] and detailed studies are found in [2-14]. None of those systems can be explored without an adequate reaction theory. In fact, the tractional distinction between the physics of nuclear bound and nuclear continuum states becomes meaningless when approaching the limits of stability. Continuum spectroscopy is an indispensable tool for studies of dripline nuclei. A prominent case is the production and spectroscopy of the particle unstable ${ }^{10} \mathrm{Li}={ }^{9} \mathrm{Li}+n$ system and other light dripline nuclei in that mass region in $(d, p)$ and charge exchange reactions $[1,15,16]$. These microscopic methods applied in those studies are showing their full potential and predictive power especially under the extreme conditions of large charge asymmetry in exotic nuclei. Essentially the same many-body theoretical methods can be applied to strangeness carrying hypernuclei. Hypernuclear physics, however, is a demanding field because it relies to a much larger extend on theoretical studies than pure isospin systems. As an extension of our

\footnotetext{
${ }^{\mathrm{a}}$ e-mail: horst.lenske@physik.uni-giessen.de

$\mathrm{b}_{\text {supported by LOEWE }}$
}

previous work [17] we have recently reconsidered the interaction model for the full SU(3) baryon flavor octet, also under the aspect of the so-called "hyperonization puzzle" [18] connected to the observation of heavy neutron stars of two solar masses. A new demand to nuclear theory is posed by recent experiments at the FRS@GSI showing clear signals for the excitation of $\Delta_{33}(1232)$ and $N^{*}(1440)$, the Roper resonance, in heavy nuclei. The theoretical description of "resonance matter" is a new field of nuclear theory, of importance also for understanding the role of resonances in neutron star matter.

In Sec. 2, recent results on nuclear multipole response functions are discussed. Hypernuclear physics, nucleon resonances, and their role in neutron stars are the topics of Sec./,3. The results are briefly summarized in Sec. 4.

\section{New modes of excitation in charge-asymmetric nuclei}

In charge-asymmetric nuclei the excess nucleons develop a layer of proton or neutron matter outside of the $N \sim$ $Z$ core, as shown in Fig. 1 for three representative isotopic chains. The effect has been seen clearly in many self-consistent calculations to nuclear ground states, e.g. our HFB results for proton and neutron skins in the Snisotopes in Ref. [4, 5]. The excess matter in skin nuclei develops specific modes of excitations, typically seen as cluster of states at excitation energy at or close to the respective particle separation threshold. Although the strength collected in these pygmy modes accounts for only a few percent of multipole sum rules, they are of large interest because of their direct relation to the size and composition of the nuclear skin. Pygmy dipole resonance modes (PDR) have attracted in the last years large attention and have been studied quite extensively by experiment and theory. Our QRPA and multi-phonon QPM calculations [2-5] are showing the distinct character of the pygmy modes. Detailed analyses of spectral distributions 


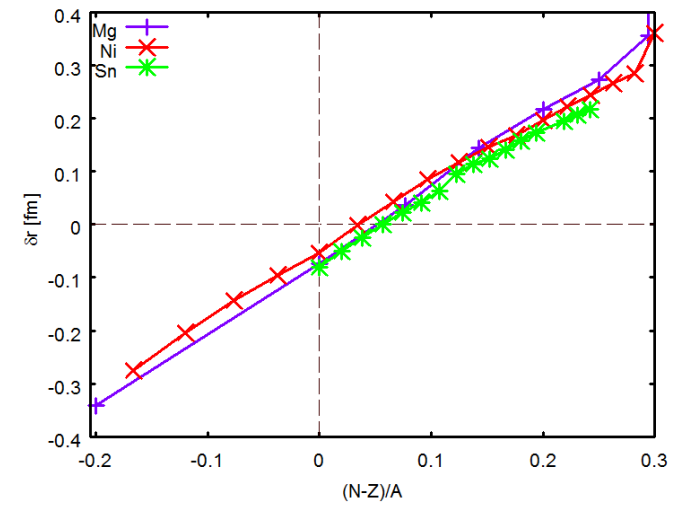

Figure 1. (Color online) Nuclear skin thickness $\delta r=\sqrt{\left\langle r_{n}^{2}\right\rangle}-$ $\sqrt{\left\langle r_{p}^{2}\right\rangle}$. HFB results for ${ }^{20-48} \mathrm{Mg},{ }^{48-82} \mathrm{Ni}$, and ${ }^{100-132} \mathrm{Sn}$ are shown as a function of the charge asymmetry.

and transition densities for isotopic and isotonic chains result in the picture that the excess nucleons vibrate with low frequency against the (almost) inert $N=Z$ core. This general feature is already present in QRPA calculations as displayed in Fig. 1. The theoretical results have been used successfully to analyse the data of different experiments, e.g. [6, 8-11, 13]. Although in many cases the overall features are already reasonably obtained by singlephonon QRPA calculations, multi-phonon effects are indispensable for a proper description of the fragmentation pattern and the total strength of pygmy modes [5].

The structure calculations are based on nuclear energy density functional $E(\rho)=T(\rho)+\frac{1}{2} \rho^{2} V(\rho)$. The interaction term is written symbolic in the reduced form as $V(\rho)$ in order to indicate that it is a superposition of one-boson exchange interactions with density dependent vertex functionals. Their structure is determined by Gmatrix in-medium interactions and three-body terms derived from the Urbana equation of state [19-21]. Single particle Hamiltonians $h_{q}$ for protons $(q=p)$ and neutrons $(q=n)$, respectively, and the residual interactions $f_{a b}$ for the various spin-isospin components are derived selfconsistently by first and second variation of $E(\rho)$, respectively,

$$
E(\rho)=E\left(\rho_{0}\right)+\sum_{q} \delta \rho_{q} h_{q}(\rho)+\sum_{a b} \delta \rho_{a} \delta \rho_{b} f_{a b}(\rho)+\cdots
$$

where $E\left(\rho_{0}\right)$ denotes the energy of the reference state, typically chosen as the ground state of a nucleus. Both $h_{q}$ and the residual interaction amplitudes $f_{a b}$ depend on the densities (or more precisely on the one-body density matrices) and include rearrangement self-energies, expressing the backflow of the system on the particle dynamics [20].

Further details of the dynamics of exotic nuclei with special focus on pygmy dynamics are discussed in these proceedings by N. Tsoneva [22]. Magnetic dipole excitations of the neutron skin in $N=50$ isotones, measured at the the High-Intensity $\gamma$-ray Source $(\mathrm{HI} \gamma \mathrm{S})$ facility at Duke university, were investigated in $[9,13]$. Our calculations led to unexpected conclusion that the pygmy $M 1$ strength
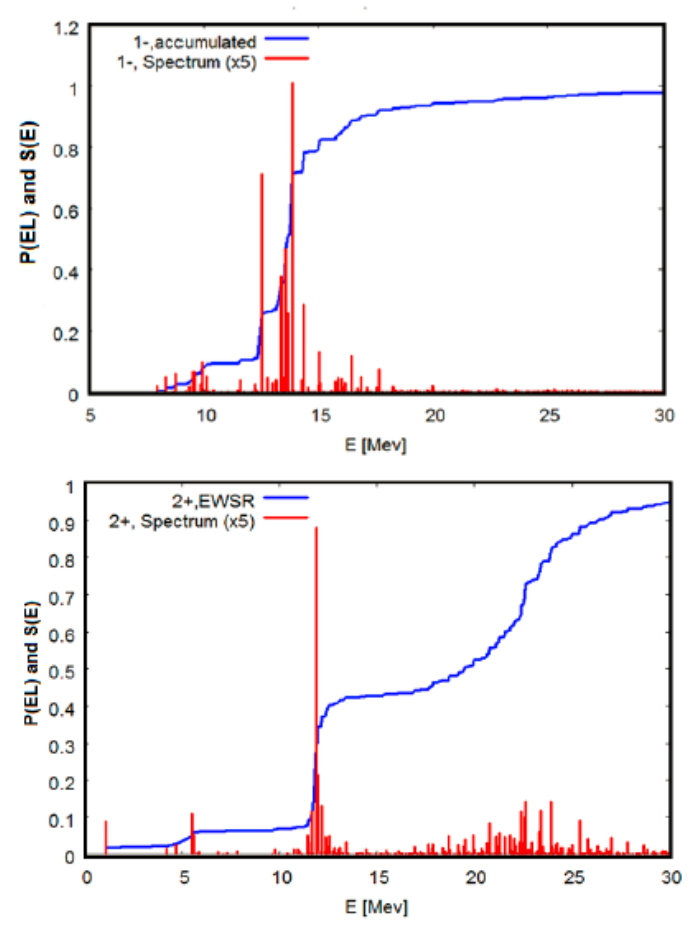

Figure 2. (Color online) QRPA dipole (upper panel) and quadrupole response (lower panel) for ${ }^{128} \mathrm{Sn}$. The accumulated excitation probability $S(E)=S_{1}(E) / S_{1}$, given by the running energy weighted multipole sum rules $S_{1}(E)=\sum_{n} E_{n} B(E L) \Theta(E-$ $E_{n}$ ), normalized to the corresponding total energy weighted sum rules $S_{1}$, and the excitation probabilities $P(E L)=E_{n} B_{n}(E L) / S_{1}$ are shown together with the energy spectra. The latter were multiplied by the indicated factor to fit the scale. For both multipolarities the onset of pygmy modes is clearly seen.

contains a large amount of orbital excitations which, however, is not identical with the well known scissors mode. As pointed out in [7] the nuclear skin naturally can be excited into any kind of multipole mode, beyond dipole modes. The pygmy quadrupole mode (PQR), predicted in that work, has been confirmed in a very recent experiment [14]. QRPA results for PQR excitations are shown also in Fig. 2.

The QPM results have recently applied very successfully in astrophysical investigations [12,23]. The model as used for beta-stable and unstable nuclei is found to provide a nearly perfect description of astrophysical reaction rates, much better than the widely used QRPA approach which, in fact, should be considered as a first order approximation. The very encouraging results indicate that our EDFbased multi-phonon theory may be used for future predictive astrophysical studies. In Fig. 3, QRPA spectral functions for charge exchange excitations in $\mathrm{Ni}$-isotopes from the proton dripline to close the neutron dripline are shown. Such results are of high relevance for nucleosynthesis reaction rates and neutrino processes in the late stages of stars and neutrons star mergers. 

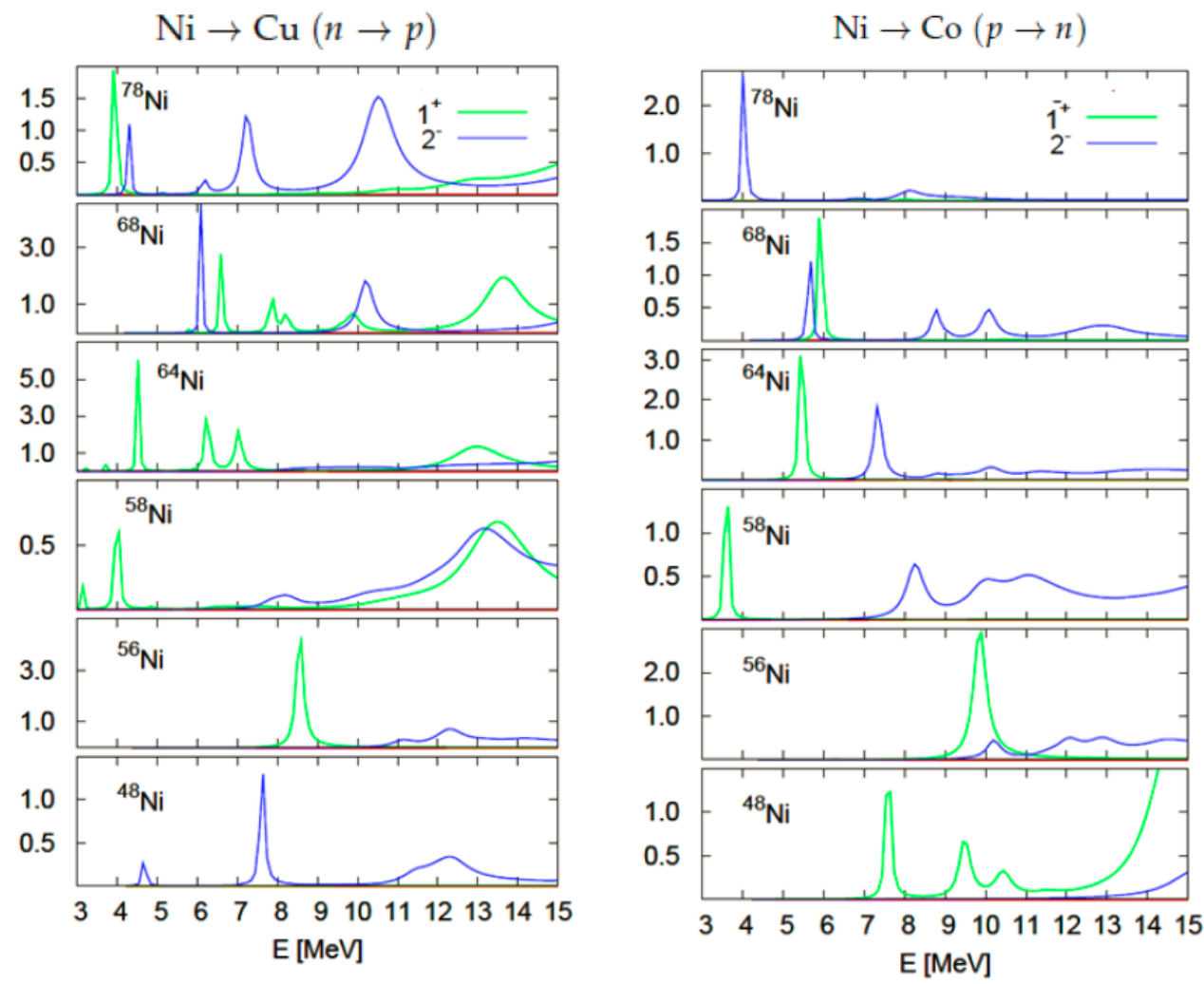

Figure 3. (Color online) QRPA multipole response functions for charge exchange excitations of the Ni isotopes. Gamow-Teller type spin-isospin flip $p^{-1} n$ and $n^{-1} p$ excitations of multiparity $1^{+}$and $2^{-}$are shown in the left and the right panel, respectively.

\section{Hyperons and resonances in nuclear matter and neutron stars}

An obvious step beyond traditional isospin nuclear physics is to extend the investigations to other SU(3) flavor sectors. Since hyperons are belonging to the same SU(3) flavor multiplet as the nucleons their inclusion is a natural extension. Thus, the full octet is included with hyperons $Y=\Lambda, \Sigma^{0, \pm}, \Xi^{0,-}$ and corresponding hypernuclei. Besides the interest in $S=-1,-2$ hypernuclei, hyperons are playing a crucial role in neutron stars. The so-called "hyperonization puzzle" in neutron star matter [18] is one of the urgent questions of neutron star physics. That question will be addressed below. Other subnuclear intrinsic degrees of freedom of baryons are accessed by the excitation of nucleon resonances. This exciting topic has been readdressed recently in experiments at FRS@GSI [24, 25] and such studies are likely to become an important topic for future experiments at FAIR.

\subsection{Hyperons in nuclear matter and neutron stars}

A caveat of extensions beyond the nucleon sector is that we know only little about the interactions of hyperons and close to nothing of resonance interactions. Various kinds of SU(3) based models for the octet baryons have been formulated and are actively worked on [26-28]. In our one boson exchange (OBE) approach we are especially interested in a unified description of interactions in free space

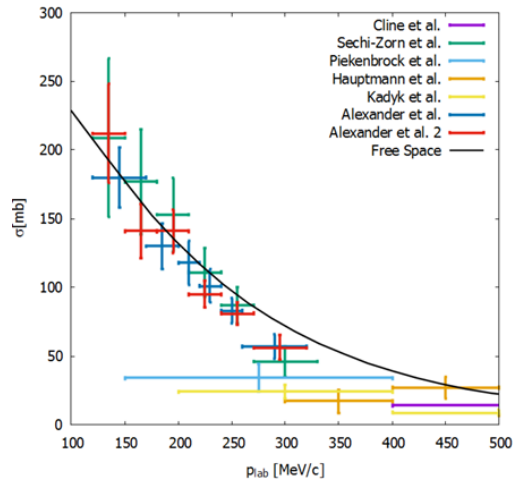

Figure 4. (Color online) Results of our SU(3) approach to $B B$ octet interactions for the $\Lambda p$ total cross section are compared to data.

and in nuclear matter with the long-term perspective of formulating a $\mathrm{SU}(3)$ density functional theory on the line of $[17,20]$. In Fig. 4, our results for the $\Lambda p$ cross section are compared to the available world data set. The baryonbaryon channels are dynamically coupled within the various multiplets of total charge $Q$ and total strangeness $S$, e.g. the $Q=0, S=-1$ multiplet formed by $\Lambda n, \Sigma^{0} n$, and $\Sigma^{-} p$. The density dependence of in-medium YN interactions is illustrated in Fig. 5 where the $\Lambda n$ and the $\Sigma^{+} p$ swave singlet scattering length $a_{s}\left(k_{F}\right)$ are displayed, evaluated inside infinite symmetric proton-neutron nuclear mat- 


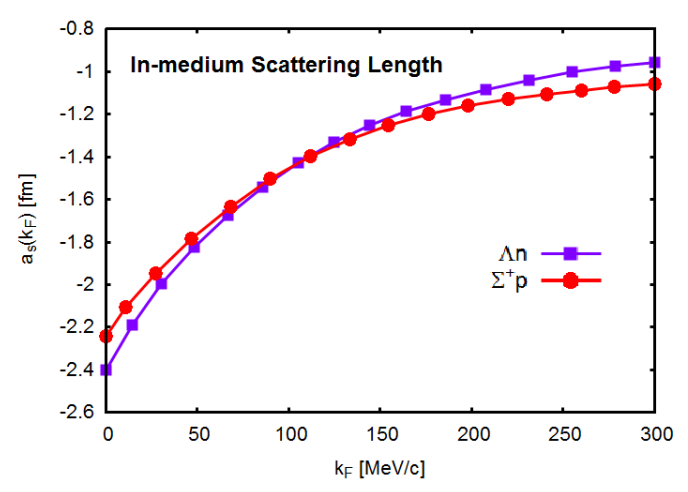

Figure 5. (Color online) Density dependence of in-medium hyperon-nucleon interactions in symmetric nuclear matter. OBE-results scattering length in the $S=-1$ channels are shown as a function of the Fermi-momentum of the background medium. $\Lambda n$ belongs to the charge-neutral triplet and is coupled to the $\Sigma^{0} n$ and the $\Sigma^{-} p$ channels.

ter at Fermi momentum $k_{F} \sim \rho^{1 / 3}$. The singlet $(S E)$ and triplet $(T E)$ s-wave scattering are in fact the leading order terms in a low-density expansion of the $N N$ interaction energy density, $V(\rho) \rightarrow \frac{3}{4} \frac{4 \pi \hbar^{2}}{M}\left(a_{S E}+a_{T E}\right)+O\left(k_{F}^{2}\right)$.

The discovery of neutron stars heavier than two solar masses in 2010 has initiated a large amount of work on the theoretical side. The approaches existing at that time were unable to reach such high masses if hyperons were included, including our own [17]. Various solutions of the so-called hyperonization puzzle have been proposed, most of them trying to suppress completely the appearance of hyperons, see e.g. [18]. As a new approach to the problem, we introduce a density dependent hyperon-vector meson interaction vertex $g_{Y v}^{*}(\rho)=g_{Y v}\left(1+h(\rho) \rho^{\beta}\right)$, where the density form factor $h(\rho)$ is chosen such that the density region encounter in hypernuclei is not affected by the additional repulsion [29]. We find an interesting hyperon shell structure. As seen in Fig. 6 hyperons appear only a small density window $\rho_{c 1}<\rho<\rho_{c 2}$ at a critical lower density $\rho_{c 1}$ and vanish again at $\rho=\rho_{c 2}$. Hence, instead of full suppression it is in fact sufficient to constrain hyperons to a limited density region. The corresponding mass-radius relation is shown in Fig. 7.

The origin of this peculiar density dependence is under investigation. Most likely, it can be understood to originate from effective three-body forces introduced by virtual excitations of the Dirac-sea at high density. In Dirac-Brueckner calculations with Dirac-sea polarization [30] we found indeed a strong increase of the vector repulsion at higher densities, introduced dynamically by the so-called Z-graphs, describing the coupling of positive energy states to excitations of the Dirac-sea. Such effects are likely to be strongly enhanced in the high density region in the interior of a neutrons star.

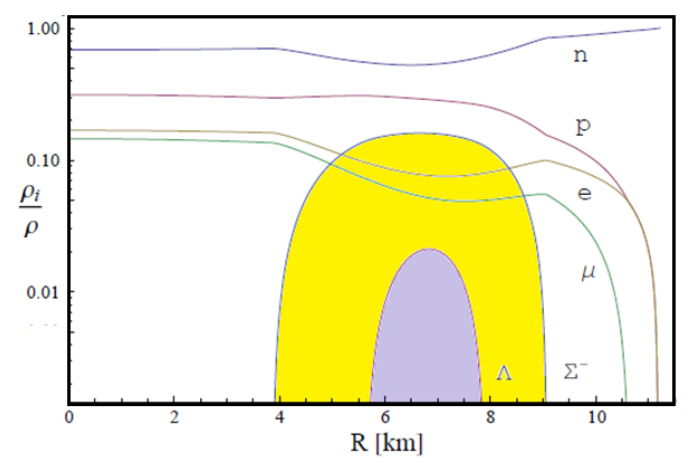

Figure 6. (Color online) Neutron star baryon fractions obtained with density dependent hyperon-vector meson coupling constants. The partial occupation numbers are shown as a function of the neutron star radius. The hyperons are confined to a spherical shell $R_{1}<r<R_{2}$. Only $\Lambda$ and $\Sigma^{-}$hyperons contribute while the other octet hyperons are completely suppressed.

\subsection{Resonances in nuclear matter}

With nucleon resonances the picture is further extended into higher baryon multiplets. The first excited state of the nucleon is the $\Delta_{33}(1232)$ resonance with mass $M_{\Delta}=$ $1232 \mathrm{MeV}$. That state is the lowest member of the SU(3) decuplet, involving also excited states $Y^{*}$ of the $S=-1,-2$ octet hyperons and the $\Omega$ baryon with strangeness $S=-3$. The decuplet baryons are having much shorter life-times $\tau_{1 / 2} \sim 10^{-24}$ sec because they decay easily by strong interaction. First attempts to produce and study the $\Delta_{33}$ in nuclei were made already some time ago at several laboratories, especially at the SATURNE facility at Saclay [31]. The interest in sub-nuclear degrees of freedom is manifold. For instance, three-body interactions which are material for nuclear saturation are mainly due to intermediate excitations of $N^{*}$ resonances. The Delta-resonance plays an important role in understanding magnetic moments and transitions and the long-standing quenching problem of

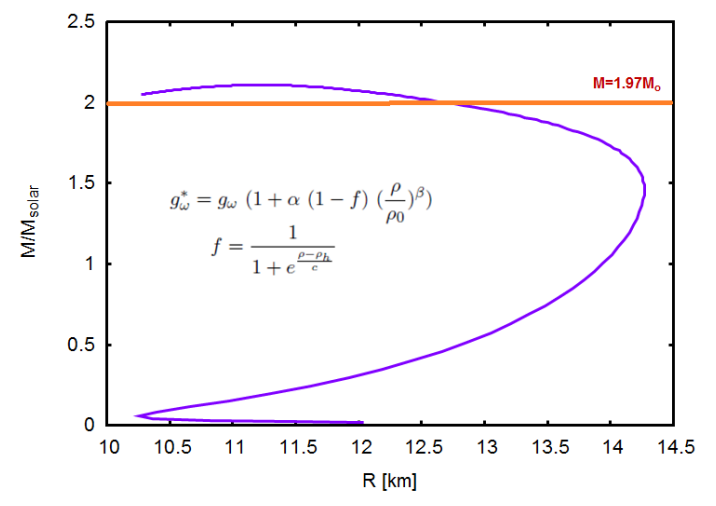

Figure 7. (Color online) Neutron star mass-radius relation by solving the TOV equations with density dependent hyperonvector meson coupling constants, confining hyperons to a shell $R_{1}<r<R_{2}$ 


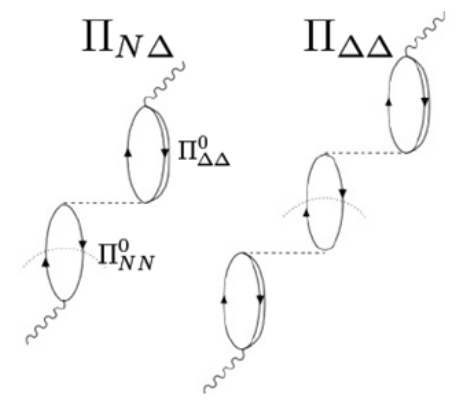

Figure 8. Diagrammatic structure of the RPA polarization propagators. For brevity we use the notation $\Pi_{N N} \equiv \Pi_{N N, N N}$ and $\Pi_{\Delta \Delta} \equiv \Pi_{N \Delta, N \Delta}$ etc.

charge-exchange Gamow-Teller excitations. Charged current high energy neutrino-nucleus interactions proceed to a large part through $N^{*}$ excitation. Last but not least, subnuclear degrees of freedom are naturally excited in dense baryonic matter as found in neutron stars [32, 33].

Experimental studies of resonance excitation in nuclei rely on beams of sufficiently high energies and appropriate detectors. The GSI facilities are providing these conditions. In a recent FRS experiment [25], the feasibility of such measurements was demonstrated very successfully in heavy ion single charge exchange reactions with relativistic beams of stable and unstable Sn-isotopes on a variety of targets, ranging from carbon to lead. The investigations will be continued and intensified in the future at the Super-FRS which is worldwide the only facility for such experiments. On the theoretical side, the renewed interest in that topic has initiated corresponding activities by the Giessen and Coimbra groups [34]. Theoretically, the excitation of $N^{*}$ resonances in nuclear matter is well covered by an appropriate extension of RPA-theory, see e.g. [35, 36]. The well known nucleonic particle-hole excitations are supplemented by nucleon hole-resonance particle $\left(N^{-1} N^{*}\right)$ configurations. In this extended configuration space the nuclear polarization propagator becomes a matrix where each resonance increases the dimension of the configuration space by one. As an example we consider the $N^{-1} N$ and $N^{-1} \Delta$ case. We denote the bare, non-interacting particle-hole tensor by $\Pi^{(0)}$, the interacting RPA tensor by the Dyson-equation $\Pi=\Pi^{(0)}+\Pi^{(0)} V \Pi$ and the particle-hole interaction by $V$. The RPA polarization tensor $\Pi$ as well as $\Pi^{0}$ and $V$ have a formal two-by-two structure,

$$
\begin{aligned}
\Pi & =\left(\begin{array}{ll}
\Pi_{N N, N N} & \Pi_{N N, N \Delta} \\
\Pi_{N \Delta, N N} & \Pi_{N \Delta, N \Delta}
\end{array}\right) \\
\Pi^{(0)} & =\left(\begin{array}{cc}
\Pi_{N N, N N}^{(0)} & 0 \\
0 & \Pi_{N \Delta, N \Delta}^{(0)}
\end{array}\right) \\
V & =\left(\begin{array}{ll}
V_{N N, N N} & V_{N N, N \Delta} \\
V_{N \Delta, N N} & V_{N \Delta, N \Delta}
\end{array}\right)
\end{aligned}
$$

describing the excitations and propagation of the $N^{-1} N$ and the $N^{-1} \Delta$ configurations in the nuclear medium. The
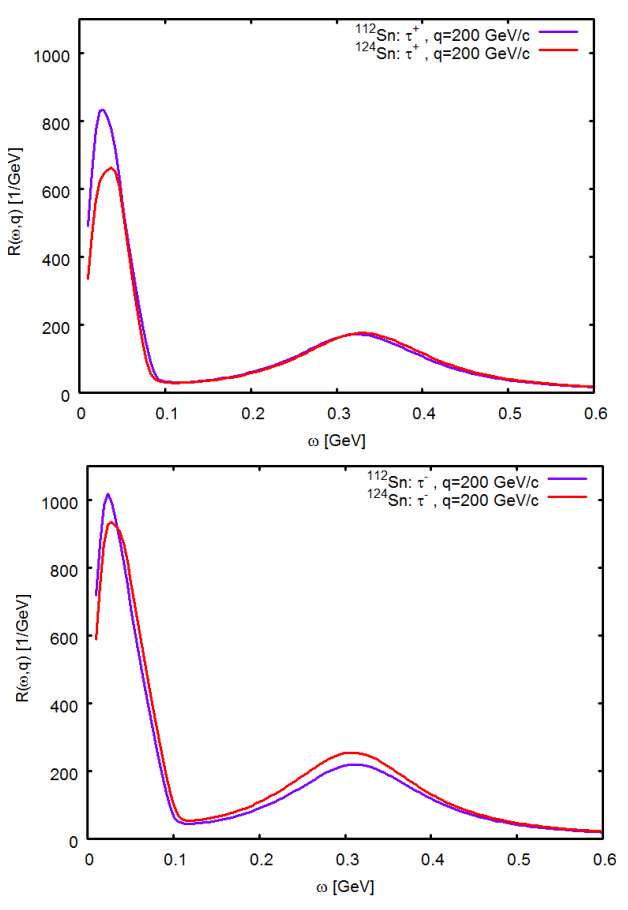

Figure 9. (Color online) Charged current response functions in ${ }^{112,124} \mathrm{Sn}$ as a function of the excitation energy $\omega$ and fixed momentum transfer $q$. The quasi-elastic peak at low excitation energies and the $\Delta$ peak at higher energies are clearly visible.

diagrammatic structure of the polarization tensor is indicated in Fig. 8. The solution of the RPA Dyson-equation leads in the higher order terms to a mixture of the bare $N^{-1} N$ and $N^{-1} \Delta$ excitations. Hence, the two components affect each other and the RPA approach accounts for these mutual modifications in a proper way. The renormalization experienced by the quasi-elastic response through the coupling to the resonance region can be expressed formally in terms of susceptibility tensor,

$$
\Pi_{N N, N N}(\omega, q)=\chi_{N \Delta}(\omega, q) \tilde{\Pi}_{N N, N N}(\omega, q),
$$

where $\tilde{\Pi}_{N N, N N}$ is the RPA polarization tensor without coupling to the resonance excitations.

Response functions for a given transition operator $T_{\alpha}$ are obtained by

$$
R_{\alpha}(\omega, q)=-\frac{1}{\pi} \operatorname{Im}\left(\left\langle T_{\alpha}^{\dagger} \Pi(\omega, q) T_{\alpha}\right\rangle\right)
$$

where the energy and momentum transfer are indicated by $\omega$ and $q$, respectively, and the brackets denote the ground state expectation value. Response functions for the charged current operators $\tau^{ \pm}$acting on ${ }^{112,124} \mathrm{Sn}$ are displayed in Fig. 9.

In the heavy ion charge exchange reactions, performed at the FRS, both isospin directions are recorded simultaneously. At the FRS-energies of 1-2 AGeV the reactions are well described by eikonal and Glauber theory, as discussed at this meeting by Vidaña [34]. 


\section{Summary}

Various aspects and realizations of exotic nuclear matter were discussed. New modes of excitation in chargeasymmetric nuclei with either a proton- or a neutronexcess were discussed in terms of QRPA and QPM theory. It is worthwhile to emphasize the crucial role played by self-consistency for the proper description of those systems also in experimentally yet unexplored mass regions. In our approach this is achieved by using the methods of density functional theory to derive single particle properties and residual interactions consistently by first and second variation with respect to the various spin-isospin densities. Nuclear spectra are described by QRPA and QPM methods. The theoretical results have been applied extensively in nuclear spectroscopy and in astrophysical studies. Extensions of the nuclear structure approach into the region of hyperons and nucleon resonances have been discussed. Interactions in the SU(3) flavor octet were derived by a newly formulated one boson exchange model, allowing to describe in-medium baryon-baryon interactions. A new solution to the hyperonization puzzle in neutron stars was presented by introducing a repulsive density dependence in the hyperon-vector meson vertices. The role of nucleon resonances for our understanding of nuclear properties was mentioned. The theoretical approach to resonance excitations in nuclei was derived by extending the RPA description of nuclear spectra into the region of resonance of $N^{-1} N^{*}$ configurations.

\section{Acknowledgements}

Supported in part by Helmholtz International Center for FAIR, GSI Darmstadt, BMBF, contract 05P12RGFTE, DFG, grant Le $439 / 9$.

\section{References}

[1] H. Lenske, S.E.A. Orrigo, and N. Tsoneva, Prog. Part. Nucl. Phys. 66, 368 (2011)

[2] N. Tsoneva, H. Lenske, and Ch. Stoyanov, Nucl. Phys. A 731, 273 (2004)

[3] N. Tsoneva, H. Lenske, and Ch. Stoyanov, Phys. Lett. B 586, 213 (2004)

[4] N. Tsoneva and H. Lenske, Prog. Part. Nucl. Phys. 59, 317 (2007)

[5] N. Tsoneva and H. Lenske, Phys. Rev. C 77, 024321 (2008)

[6] R. Schwengner et al., Phys. Rev. C 78, 064314 (2008)

[7] N. Tsoneva and H. Lenske, Phys. Lett. B 695, 174 (2011)

[8] A.P. Tonchev, S.L. Hammond, J.H. Kelley, E. Kwan, H. Lenske, G. Rusev, W. Tornow, and N. Tsoneva, Phys. Rev. Lett. 104, 072501 (2010)
[9] G. Rusev et al., Phys. Rev. Lett. 110, 022503 (2013)

[10] R. Schwengner et al., Phys. Rev. C 87, 024306 (2013)

[11] B. Özel-Tashenov et al., Phys. Rev. C 90, 024304 (2014)

[12] N. Tsoneva, S. Goriely, H. Lenske, and R. Schwengner, Phys. Rev. C 91, 044318 (2015)

[13] Krishichayan, M. Bhike, W. Tornow, G. Rusev, A.P. Tonchev, N. Tsoneva, and H. Lenske, Phys. Rev. C 91, 044328 (2015)

[14] L. Pellegri et al., Phys. Rev. C 92, 014330 (2015)

[15] S.E.A. Orrigo et al., Phys. Lett. B 633, 469 (2006)

[16] S.E.A. Orrigo and H. Lenske, Phys. Lett. B 677, 214 (2009)

[17] C. Keil, F. Hofmann, and H. Lenske, Phys. Rev. C 61, 064309 (2000)

[18] J. Zdunik and P. Haensel, Astronomy and Astrophysics 551, A61 (2013); M. Fortin, J.L. Zdunik, P. Haensel, and M. Bejger, Astronomy and Astrophysics 576, A68 (2015)

[19] F. Hofmann and H. Lenske, Phys. Rev. C 57, 2281 (1998)

[20] H. Lenske, Springer Lecture Notes 641, 147 (2004)

[21] A. Fedoseew and H. Lenske, Phys. Rev. C 91, 034307 (2015)

[22] N. Tsoneva, these proceedings.

[23] R. Raut et al., Phys. Rev. Lett. 111, 112501 (2013)

[24] J. Vargas, J.Benlliure, and M. Caamanõ, NIM A 707, 16 (2013)

[25] J. Benlliure et al., JPS Conf. Proc. 6, 020039 (2015)

[26] Th.A. Rijken, V.G.J. Stoks, and Y. Yamamoto, Phys. Rev. 59, 21 (1999)

[27] J. Haidenbauer, U.-G. Meissner, A. Nogga, and H. Polinder, Lect. Notes Phys. 724, 113 (2007)

[28] Y. Fujiwara, Y. Suzuki, and C. Nakamoto, Prog. Part. Nucl. Phys. 58, 439 (2007)

[29] J. Wilhelm, Thesis, Univ. Giessen, 2014; J. Wilhelm and $\mathrm{H}$. Lenske, in preparation.

[30] F. de Jong and H. Lenske, Phys. Rev. C 58, 890 (1998)

[31] C. Ellegard et al., Phys. Rev. Lett. 50, 1745 (1983)

[32] A. Drago, A. Lavagno, G. Pagliara, and D. Pigato, arXiv: 1407.2843, 2014

[33] T. Schürhoff, S. Schramm, and V. Dexheimer, Astrophys. J. Lett. 724, L74 (2010)

[34] I. Vidaña, these proceedings.

[35] M. Martini, M. Ericson, G. Chanfray, and J. Marteau, Phys. Rev. C 80, 065501 (2009)

[36] J. Nieves, I. Ruiz Simo, and M.J. Vicente Vacas, Phys. Rev. C 83, 045501 (2011) 